\title{
Erratum to: Progressive changes of orexin system in a rat model of 6-hydroxydopamine-induced Parkinson's disease
}

\author{
Long-Biao Cui ${ }^{1,2} \cdot$ Bo-Wei $\mathrm{Li}^{1,2} \cdot$ Xiao-Hang Jin ${ }^{1}$.
}

Lin $\mathrm{Zhao}^{3} \cdot$ Juan Shi $^{1}$

Published online: 22 June 2016

(c) Shanghai Institutes for Biological Sciences, CAS and Springer Science+Business Media Singapore 2016

Erratum to: Neurosci. Bull. October 1, 2010, 26(5): 381-387. http://www.neurosci.cn DOI: $10.1007 / \mathrm{s} 12264-010-0410-9$

In the original publication of the article Fig. $4 \mathrm{C}$ has been incorrectly published due to mislabelling of the image file in the primary record. The correct figure is provided in this
The online version of the original article can be found under doi:10.1007/s12264-010-0410-9.

\section{Juan Shi}

shixjuan@fmmu.edu.cn

1 Department of Human Anatomy, Histology and Embryology, The Fourth Military Medical University, Xi'an 710032,

China

2 School of Aerospace Medicine, The Fourth Military Medical University, Xi' an 710032, China

3 Department of Human Anatomy, Medical College of Yan'an University, Yan'an 716000, China erratum. The authors sincerely apologize for this inadvertent mistake. This correction does not alter the conclusions of this article.

The correct Fig. 4C.
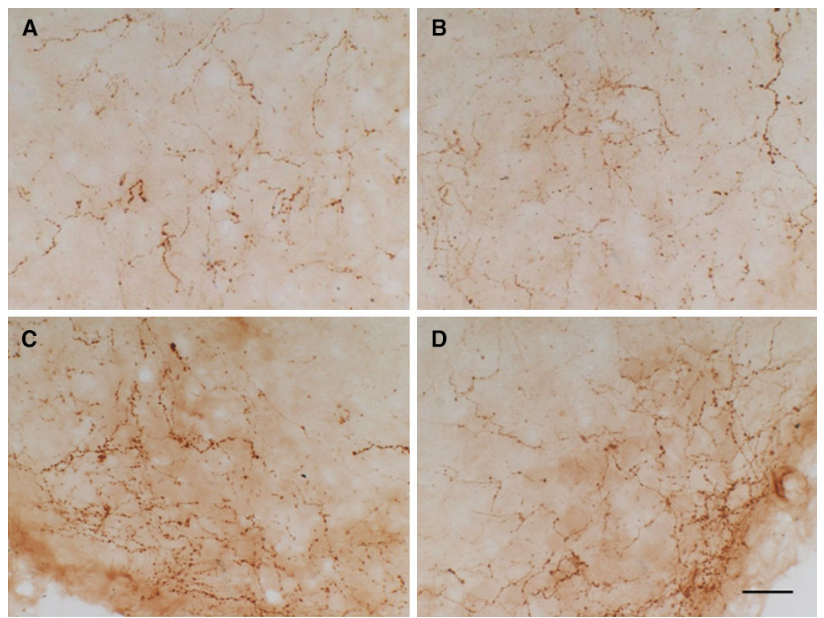

Fig. 4 Immunohistochemical staining of orexin-A-containing fibers in TN of control $(\mathbf{A}, \mathbf{C})$ and $\mathrm{PD}(\mathbf{B}, \mathbf{D})$ rats at day 49 post operation. A, B: dorsal tuberomammillary nucleus; C, D: ventral tuberomammillary nucleus. Scale bar, $50 \mu \mathrm{m}$. 